\title{
TRES BIBLIOTECAS JESUITAS EN PUEBLOS DE MISION: BUENAVISTA, PAILA Y SANTA ROSA, EN LA REGION DE MOXOS
}

POR

CATALINA ROMERO ROMERO

\section{LAS MISIONES DE MOXOS}

La amplia vertiente que une las cordilleras andinas a las llanuras amazónicas tiene su mayor extensión a la altura del sur peruano y de la Bolivia actual. Este sector corresponde a los cursos superiores de los ríos Madre de Dios, Beni, Mamoré y Guaporé o Iténes (fronterizo con Brasil) que convergen todos para formar el río Madeira, uno de los más potentes afluentes de la orilla derecha del Amazonas.

Con características homogéneas, los jesuitas tuvieron enclavadas en estos territorios orientales bolivianos dos florecientes misiones la llamada de Moxos, dependiente de la provincia jesuítica peruana y la de Chiquitos, fundada y sostenida por la provincia jesuítica del Paraguay.

Las primeras noticias sobre este área se deben a la correspondencia (1) de los jesuitas. En sus cartas la descripción del territorio tendrá un denominador común: zona de relieve accidentado, humedad permanente, flora y fauna exhuberante y gran multitud de etnias dispersas por ella. Estas características físicas de la región van a condicionar toda la actuación de los misioneros, por ello hasta finales del siglo XVI, 1570, se encontrarán en éstas márgenes no controladas un cierto número de clérigos y frailes que actuarán por su propia cuenta.

Trabajo efectuado dentro del Programa de Investigación PB87-0375 financiado por el Ministerio de Educación y Ciencia.

Siglas UTILIZADAS:

AHN: Archivo Histórico Nacional, Madrid.

RAH: Real Academia de la Historia, Madrid.

(1) Cartas del 9-XI-1668 y 10-IX-1680 en Rubén VARcias Ugarte: Historia de la Compañia de Jesilis en el Perú. Burgos, 1964. 
Las primeras incursiones de los jesuitas en territorio de Moxos serán a través de Santa Cruz de la Sierra, 1580, un camino al parecer bastante largo, según demuestran más tarde los misioneros, Antonio Orellana y José de Vega en 1688, al trazar una nueva ruta, más corta y breve desde Cochabamba.

La entrada en esta región fue un estímulo constante para los jesuitas de Santa Cruz desde el primer momento de su implantación en ella, probablemente contagiados como muchos otros de los mitos del "Reino de los Muxu» de los Incas, "Paititi» o "Eldorado" (2), de los guaraníes. Estos mitos que impregnan los relatos de la época explican en gran parte el proceso colonizador de este área oriental.

La conquista de las tierras de Moxos fue iniciada en 1594 por el gobernador de Santa Cruz de la Sierra, Lorenzo Suárez de Figueroa al que acompañaron en esta primera expedición los jesuitas Diego de Samaniego y Jerónimo de Andión, madrileño de unos 40 años que había ingresado en la Compañía de Lima en 1585 y misionado en la residencia cruceña; pero en 1595 con la muerte del gobernador (3) se suspendió la empresa.

En 1601, el superior crucense de los jesuitas, Diego Martínez, resumía así el aporte de esta expedición:

En este tiempo supieron los soldados la muerte del gobernador don Lorenzo Suárez de Figueroa, y como les faltó la cabeza, acabadas las aguas (marzo de 1596) (4), ya que tenían la noticia en las manos, se volvieron todos los soldados, y de camino hicieron lo que suelen de maldades, matando indios y cautivando a todos los que pudieron haber a las manos. Un cacique que trajeron cautivo, nos contaba 160 pueblos en muy poca distancia. De esta conquista se tiene grandes esperanzas de muchos indios y riquezas, y que se espera gran descubrimiento de provincias, así hacia las cordilleras de Perú desde esta parte del

(2) El mito de Eldorado fue contagiado a los españoles por los guaraníes. Nuflo de Chavez, fundador de Santa Cruz de la Sierra nos díce que ésta nació como simple base provisional para las expediciones posteriores. El capellán jesuita de la expedición que costea con el gobernador de Santa Cruz, Lorenzo Suárez de Figueroa nos dice: "Vinimos siempre ribera el río Guaypay... por tener noticia... de los Moxos o Paytitin o Candire como acá le llaman" (1595). En: P. Pastell y F. Mateos: Historia de la Compañia de Jesús en la Provincia del Paraguay. Madrid, 1912-49, 8 vols.

(3) Según los cronistas jesuitas, de melancolía por no haber conseguido Eldorado.

(4) Durante los meses de noviembre a marzo esta zona era intransitable por ser la época de lluvias torrenciales. 
Guaypay, como de la otra parte del río hacia Brasil, donde dicen esta la laguna de los moxos (5).

La primera impresión de la nación Moxa la expresó el padre Andrés Ortiz en una carta de 1595 al provincial Juan Sebastián:

Es ésta una gran provincia, poblada de gente vestida y política y que tiene y se sirve de plata, de que ha muchos años se tiene grande noticia; los que poblaron la ciudad de Santa Cruz de la Sierra no tuvieron por principal objeto el hacer allí asiento, sino que aquella ciudad y las demás fuesen escala para el descubrimiento que pretendían (6).

Se volvieron a repetir las incursiones hacia 1602, con la expedición de J. Mendoza Mate de Luna con resultados escasos.

El 2 de octubre de 1607, el gobernador Martín de Almendras Holguín, piensa que lo más acertado es confiar a particulares la empresa, para no arriesgar tantos hombres y dinero del Estado; por eso en ese mismo año entregaba a Gonzalo de Solís Holguín, y a los suyos la encomienda de Moxos, durante dos vidas, con la condición de fundar en su territorio una ciudad con la advocación de la Santísima Trinidad y de adoctrinar a sus habitantes. Pero ésto también fracasó.

La conquista de estas tierras se emprenderá nuevamente hacia 1662, cuando el hermano Juan de Soto baja por el Guapay hacia las primeras etnias moxas; y posteriormente en 1668 los jesuitas Soto, José Bermudo y Julián Aller, entrarán definitivamente en tierras de Moxos, sobre todo para el estudio de la lengua; pero hasta 1682 no se fundará la primera reducción. Tuvieron que pasar quince años de aprendizaje, adaptación y penosas experiencias hasta lograr el paso decisivo. Éste llegará hacia 1674 con el hermano José del Castillo y al año siguiente con los padres Pedro Marbán (7), Cipriano Barace y José Bermudo, enviados por el visitador de la provincia peruana Hernando Cavero, el mismo que había comenzado años atrás la conquista del Orinoco.

La Provincia Peruana de la Compañía de Jesús, no acababa

(5) Relación del padre Diego Martínez al provincial de Chuquisaca (24 de abril de 1601). En: Francisco Mateos (ed.): Historia General de la Compañia de Jesús en la Provincia del Perú, Madrid, 1944.

(6) Carta del padre Andrés Ortiz al provincial padre Juan Sebastián. En: Antonio Egaña: Historia de la Iglesia en la América Española, Madrid, 1966.

(7) Recogió los primeros datos que después compiló para realizar la édición de un vocabulario de la lengua moxa. 
de ver claro la posibilidad de aquellas misiones; ya que no existía ninguna reducción estable después de tanto esfuerzo. Por ello en 1678 y 1681, los provinciales de los jesuitas del Perú deciden enviar como visitadores a los padres Luis Sotelo y Lituria respectivamente para que tomaran una decisión sobre la viabilidad de la iniciativa. Estos padres después de analizar las circunstancias y posibilidades dieron luz verde al proyecto.

Así los jesuitas lograron establecerse entre los moxos de un modo estable y fijo desde 1681. Los padres Cipriano Barace (8) y Baltasar Espinosa fundaron la misión de San Pedro - con pueblos baure y moxo-; Nuestra Señora del Loreto en 1681, considerada la primera reducción, que prosperó pronto y se introdujo por primera vez ganado vacuno en la región del Beni; Trinidad (1686) fundada por Barese donde permaneció 27 años, hasta su muerte en 1702; San Javier, Exaltación, San Ignacio; Santa Ana -de etnia mobina-; San Borja; Reyes - con grupos moxo y baure-,... que se amplían a principios del siglo XVIII por la acción del padre Lorenzo Legarda con los pueblos de Magdalena, Concepción; San Joaquín; Buenavista o Desposorios de Nuestra Señora; la nueva San Pedro, fundada por Estanislao Arlet (9), en la ribera derecha del Mamoré (capital de Moxos a finales del siglo XVIII y residencia del superior-; San Martín (10) y San Pablo, fundadas por Francisco Boryne, donde vivió de 1696 a 1722, promoviendo la edificación de las misiones así como la ganadería y la música.

Según Josep M. Barnadas, en un artículo de 1984 titulado Las reducciones jesuiticas de Mojos (11), la expansión jesuita en este territorio, mostraria las siguientes etapas:

Primera (1682-1700: Sobre el eje el Mamoré y las Pampas de occidente se fundan las reducciones de

1. Loreto (1682), al oeste del Mamoré.

2. Trinidad (1687), al este del Mamoré.

3. San Ignacio (1689), al oeste del Mamoré.

(8) Fue el descubridor de la ruta más corta entre los llanos amazónicos y la región andina de Bolivia, así como el introductor de la industria pecuaria con ganado en Moxos; también se destacó como profesor de música y artesanía.

(9) Fue rector del colegio jesuita de La Plata, murió en el colegio de Potosí en 1717.

(10) En esta misión residió el padre Francisco Javier Eder, nacido en Hungría en 1727 y que después de la expulsión (1767) ya en su patria natal se dedicó a la redacción del libro: Breve descripción de las reducciones de Moxos en el reino del Perú (1772).

(11) En Historia Boliviana. Cochabamba (Bolivia), 1984, págs. 135-166. 
4. San Francisco Javier (1691), al este del Mamoré.

5. San José (1691), al oeste en las llanuras a unas 70 leguas de Cochabamba.

6. San Francisco Borja (1693), al oeste en las riberas del río Manaquí.

7. Desposorios de Nuestra Señora (A), 1694.

8. San Miguel (A), 1696.

9. San Pedro, 1697.

10. San Luis, 1698.

Segunda (1700-1720): en la que prosigue la expansión por los pampas y se lleva a cabo la penetración en Baures

11. San Pablo, 1703.

12. Santa Rosa (A), 1705.

13. Concepción, 1708.

14. Exaltación, 1709.

15. San Joaquín, 1709.

16. Tres Santos Reyes, 1710.

17. San Juan Bautista, 1710.

18. San Martín, 1717.

19. Santa Ana, 1719.

20. Santa María Magdalena, 1720.

Tercera (1720-1750): durante la cual se consolida la penetración en Baures hasta las orillas del Iténes y se pone un hito de contacto con Santa Cruz de la Sierra

21. Desposorios de Nuestra Señora, 1723.

22. San Miguel (B), 1725.

23. Patrocinio de Nuestra Señora, 1730.

24. San Nicolás, 1740.

25. Santa Rosa (B), 1743.

26. San Simón, 1744.

Si tenemos presente este esquema podemos afirmar que a principios del siglo XVIII el territorio de Moxos había adquirido sus contornos definitivos, ocupando el extenso curso alto de los ríos Mamoré, Iténes o Guaporé y Beni, afluentes del Madeira; los pueblos anteriormente citados se extendian en forma de cruz formando tres regiones naturales, la Central, a lo largo del Mamoré, la de los Baures hacia oriente, y la de los Pampas hacia poniente hasta el río Beni. Algunas de ellas rebasaron el margen 
derecha del río Iténes, adentrándose en territorios que actualmente pertenecen a Brasil.

\section{LAS MISIONES DE MOXOS EN EL SIGLO XVIII}

Las misiones de Moxos, enclavadas en territorio de la audiencia de Charcas, y pertenecientes al obispado de Santa Cruz de la Sierra, aunque las sostenía, como todas las casas de jesuitas de la actual Bolivia, menos Tarija, la provincia jesuítica peruana, en la primera mitad de este siglo no sufrieron alteraciones notables, fuera de la destrucción del pueblo de San Miguel y el traslado de Santa Rosa a la margen izquierda del Iténes.

Además viendo la importancia de la navegación fluvial para la vida interna de las misiones, los jesuitas habían creado sus puertos urbano-misionales, como en San Simón, próximo al Guaporé en 1742 y al año siguiente en Santa Rosa, donde después se situó el Fuerte del Príncipe de Beyra, sobre la ribera derecha del Iténes, hasta que los portugueses expulsaron a los misioneros españoles, por juzgar que éstos les impedían el paso a la zona lusitana y como consecuencia directa del tratado de Límites.

Hacia 1713, las misiones de Moxos (12) contaban con 17 pueblos y más de 30.514 indios, asistidos por 37 misioneros, como lo testifica el obispo de Santa Cruz de la Sierra, Jaime Mimbela, quien entró a hacer la visita pastoral en 1712. El procurador Francisco de Rotalde obtuvo del Consejo de Indias en 1716, que se concediera a los misioneros el sínodo o subsidio pecuniario de que hasta entonces habían carecido, costeándose todos los gastos con las limosnas de las ciudades del Perú.

A mediados del siglo XVIII, los jesuitas habían consolidado sus misiones de Moxos, empleando el sistema de "reducciones», como modelo de concentración poblacional y de la evangelización indirecta a través de grupos misionales que penetraban en la selva para persuadir a etnias desconocidas, así como con la prohibición de entrada a todos los ajenos a ellas, especialmente después de una visita esclavista del gobernador de Santa Cruz en 1700.

(12) Breve noticia en que se hallan el año 1713 las misiones de infieles que tiene a su cargo la Provincia del Perú, de la Compañia de Jesís en las provincias de moxos. s.l., s.f. (16 págs.). 
Hacia 1764 se contaban 20 misiones (13), a cargo de 46 sacerdotes y coadjutores, con cerca de $\mathbf{4 0 . 0 0 0}$ habitantes de las siguientes etnias:

- Moxa, en Loreto, Trinidad, San Javier, San Ignacio.

- Baure, en Concepción, San Joaquín, San Martín y San Nicolás.

- Mure, en San Simón.

- Mobina, en Santa Ana, San Borja.

- Caniciana en San Pedro.

- Cayubaba, en Exaltación.

- Itonama en Magdalena.

- Maracam en Reyes.

Y hacia 1768, según el Informe del Obispo de Santa Cruz de la Sierra, Francisco Herboso, al virrey del Perú, don Manuel Amat (14), no había variado mucho:

La provincia peruana de la Compañía de Jesús no tenía más misiones que la de moxos, que con las dos de Buenavista y Santa Rosa inmediata a esta ciudad de Santa Cruz, son 17 y conducía desde Europa cada seis años a'gran porción de sujetos para proveerlas porque son pocos los que pueden permanecer dilatado tiempo allí, por el clima excesivamente caluroso y húmedo.

\subsection{La organización socioeconómica de las Misiones}

La principal actividad económica (15) de las reducciones estaba basada en la elaboración de azúcar y melaza en trapiches; en la ganadería, con la cría de ganado vacuno, tanto para la alimentación como para la exportación de sebo; en la agricultura: cultivo de yuca, plátanos y maíz - que se cosechaba dos o tres veces al año-; en la explotación de los recursos forestales de la zona: el jacarandá, el incienso, la copayba, la maría y el cedro. Estas maderas se usaban para levantar cabañas, fabricar muebles,

(13) Santa Rosa la Nueva (abandonada); La Exaltación; Santa Ana; San Pedro; San Javier; La Trinidad; Loreto; Los Deposorios o Buenavista; San Ignacio; San José; San Borja; San Pablo; Los Reyes; San Nicolás; San Simón; San Martí; San Joaquín; Concepción; San Miguel y Magdalena.

(14) Pastell, [2] (1912-49), pág. 1.291.

(15) David Bı.ock: "La visión jesuítica de los pueblos autóctonos de mojos (1667-1700)". En: Historia Boliviana, La Paz., 1986. 
troncos, embutidos, molduras, herramientas y canoas, además de bálsamos, aceites, gomas y resinas de variado uso.

Otras de las actividades principales de los indios eran la caza, pesca y la fabricación con la melaza del "guarapo», bebida que juntamente con la carne eran objeto de "reparto de liberalidad" entre las familias de la reducción (caso del guarapo o de la carne en cuatro oportunidades al año: Navidad, Pascua, San Javier y el Corpus) o a diario entre los necesitados (ancianos, viudas, huérfanos), o eventualmente como recompensa por las prestaciones laborales recíprocas.

Estas condiciones eran generales aunque cada misión tuvo algunos rasgos propios; en Loreto, el cacao era excelente pero no pudo adaptarse el algodón. Rodeada de pantanos y aguas estancadas, el pueblo se vio siempre sometido a fiebres palúdicas; en San Pedro y San Javier, caña de azúcar y café; Magdalena y Trinidad eran aptas para la caña de azúcar y el algodón, en esta última los cultivos ocupaban una explanada inundable, lejos del pueblo; Concepción, rodeada de montes, con un microclima de temperatura muy variable la agricultura fue rica y variada; San Pedro abunda el ganado y pequeñas huertas a las orillas del Mamoré.

Cada reducción constituía una unidad de producción, consumo y aprovisionamiento autónoma, ésto no impedía la creación de flujos de intercambio según las necesidades y recursos disponibles en cada una de ellas.

La vida en la misión (16) según el padre Altamirano significó para los indios: médicos y cirujanos que curan; enfermeros que los atienden; maestros que les enseñan los oficios; intructores que les guían en agricultura, les dan anzuelos para pescar, hachas para derribar árboles; bienhechores que les han traído vacas desde Santa Cruz "para su sustento», etc...

En el momento de la expulsión, 1767, el administrador gencral de todos los bienes de Moxos eran el padre Carlos Hirschko que declara las siguientes posesiones: viñedo de Callejón la Baja, haciendas de Challwain (viñedos) y Habana (cereales, vacuno) en el departamento de Cochabamba, los beneficios de las haciendas Mutakachi y San Jacinto. Asimismo las haciendas de Wawra, Umay y varias haciendas situadas en la región oriental de Chuquisaca: San Isidro, Jesús y María, Pampas del Tigre y Cuesta larga. 1979.

(16) Diego Francisco Altamirano: Historia de la misión de Moxos, La Paz, 
Además de 145.925 pesos invertidos en obras pías y capellanías.

Las misiones se financiaban con el aporte de la Provincia Peruana, especialmente en los comienzos, así en 1698 declaraban haber gastado 100.000 pesos $(17)$ y con contribuciones esporádicas y regulares del Rey. En 1716, dispone abonar 200 pesos anuales por cada jesuita activo en la misión. Asimismo se contaba con las limosnas y legados de bienhechores y con la renta anual de capitales invertidos (censos, haciendas).

La capacidad económica de las propias misiones: cera, telas, cacao, azúcar, arroz... con destino a Chuquisaca, Cochambamba, Oruro, La Paz y el Cuzco permitió tender lazos económicos que ligaron las misiones con Lima y la metrópoli, y que unos pocos misioneros europeos y posteriormente criollos crearan poblaciones importantes en una región aislada. Además, una gran flexibilidad de gestión de un patrimonio nominalmente adscrito a numerosas entidades de la Compañía, permitiéndole transferencias monetarias, crediticias, rentísticas y otras, gracias a su racionalidad económica.

Debido a las condiciones ambientales, fue necesario promover un intenso intercambio comercial entre las distintas reducciones, a pesar de que cada una tendía a la autosuficiencia económica como acabamos de ver. Así, algunas reducciones exportaban tejidos de algodón a los de Santa Cruz, a cambio de sal y caballos y en otros pueblos ceras de los montes; aunque eran tierras aptas para sembrar arroz y caña dulce, ésto sólo lo cultivan los misioneros. Además una variedad de manufacturas que por Santa Cruz sacan y que en el Alto Perú se vendían a cargo de los procuradores de la Compañía de Jesús en La Plata, Potosí, Oruro, Cochabamba y La Paz.

La Procaduría el colegio de Lima recibía del Alto Perú el dinero de moxos, necesario para el pago de los efectos ultramarinos que las misiones pedían y se les remitía desde allí. Para que este comercio quedara consolidado el 25 de julio de 1725, por real cédula de virrey del Perú, marqués de Castelfuerte, se les concede el uso de armas a los misioneros por ello le remiten 200 armas con pólvora.

(17) Pastell [2] (1912-49), vol. IV, págs. 415-416. 


\subsection{La vida cotidiana}

La organización de la vida familiar y social en las reducciones fue objeto de especial preocupación de los misioneros jesuitas. La sociedad de las misiones estaba dividida en dos grandes sectores: los artesanos de todos los oficios y los soldados encargados de las tareas inferiores. En cada poblado, la suprema autoridad era el cacique, ayudado por un alférez y dos tenientes; le seguían dos alcaldes de familia, dos alcaldes de pueblo y sobre todos ellos ejerciendo una autoridad espiritual-temporal, estaba el misionero, máximo responsable de la vida local.

La monotonía del día era rota con las celebraciones religiosas; para los adultos no era obligatoria la asistencia a misa durante la semana; sin embargo es habitual para la mayoría, sobre todo cuando se encontraban en el pueblo. El sábado se cantaba la Salve en la iglesia y el rosario por las calles, se juntaban todos los muchachos y niñas a toque de campana y se les explicaba la doctrina cristiana, era muy normal que a estas pláticas acudieran los recién casados. Nadie contraía matrimonio sin que precediera un riguroso examen de doctrina: Catecismo.

Los domingos y fiestas del año se hacía sermón al pueblo; antes del jubileo de 40 horas se multiplicaban de nuevo las pláticas a los indios para que cumplieran fructuosamente los dos preceptos: la confesión y comunión anual, y durante la Cuaresma cada semana dos veces se hacía plática a todo el pueblo con algún ejemplo, a la manera que los padres estilaban con los españoles en los pueblos del Perú.

Recibían la comunión en las fiestas del Señor, Santísima Madre, la Virgen María y en las fiestas de algunos santos. Las Pascuas y demás festividades cristianas se celebraban con gran solemnidad (18).

\subsection{El urbanismo (19)}

Las reducciones surgieron en zonas alejadas de los centros urbanos de población predominantemente española. La ubicación era elegida por los misioneros de acuerdo con las autoridades

(18) Francisco Javier EDER: Breve descripción de las reducciones de Mojos. Edición de Josep M. Barnadas. Cochabamba (Bolivia), 1985.

(19) María A. Buschiazzo: "La arquitectura de las misiones de moxos y chiquitos". En: Südamerika, Buenos Aires, 1953. 
coloniales, tomando en cuenta factores como la proximidad de ríos o lagunas, accesos rápidos a las planicies de pastos, posibilidad de comunicaciones. Además debería tenerse en cuenta, que el lugar elegido fuera agradable a la población indígena, por eso muchas de las misiones fueron establecidas cerca de la morada habitual de las etnias, para atraerlos poco a poco, pero esto fue una empresa difícil como hemos visto. La sedentarización fue un gran problema para los misioneros debido a los escasos recursos humanos como materiales.

Una vez seleccionado el lugar de la reducción, los misioneros procedían a la planificación del trazado urbanístico, que tenía un esquema fijo: una plaza central, constituía el corazón del poblado en ella se erigía una cruz grande, acompañada de estatuas de la Virgen y del patrón de la reducción. Allí se encontraba la iglesia, la escuela, el cementerio, las viviendas de los misioneros, los almacenes... y otros edificios públicos, como molinos, hornos, comedores, graneros, cocinas. Sobre los lados restantes se situaban las casas de los indios, dispuestas en largas filas paralelas que partían desde el centro a la periferia de la reducción. Por ejemplo en 1684, los jesuitas Pedro Marbán (superior), José de Vega el hermano Antonio Fernández y el donado Antonio Carrillo, escogen un paraje en los llanos del norte, sobre los ríos Guaypay y Mamoré, para fundar la misión Nuestra Señora del Loreto, trazan la plaza principal y las calles convergentes a la misma, comienzan a fabricar una amplia iglesia de adobe, por la falta de piedra, de tres naves de 60 varas de largo por 20 de anchas, y de paredes muy gruesas (20).

Como se puede apreciar la distribución urbana y la arquitectura eran semejantes a las reducciones guaraníes, no así en cuanto a las experiencias de los jesuitas en estas tierras ya que fueron distintas de las vividas por los misioneros en territorio guaraní, no solamente por las diferencias entre los dos pueblos sino también porque la zona donde tenían enclavadas las misiones era y es hoy todavía una región aislada, tanto del resto del país como de otras áreas. Esto determinó que los misioneros no pudieran contar con la ayuda del poder seglar, cuando tenían que vérselas con tribus peligrosas pero también que la Corona no intefiriera en su trabajo como ocurrió en el caso de las guaraníticas a las que los gobernadores acudían con asiduidad para el reclutamiento de indios destinados a la defensa de la frontera o a trabajos de zapa. Otra de las diferencias con las del

(20) Egiaña [6], págs. 399. 
Paraguay, fue el gran predominio de jesuitas criollos frente al predominio de europeos en las guaraníticas.

\section{LA EXPULSIÓN}

La orden de Carlos III, ejecutada con rapidez por el gobernador Francisco de Paula Bucarelli y Ursúa, desde Buenos Aires, llegó a la Audiencia de Charcas, y de ahí a las tierras de Moxos. En agosto de 1767 estaba en la misión de San Pedro el coronel don Antonio de Aymerich, al frente de la guarnición española destacada para vigilar y contener el avance de las fuerzas portuguesas por.las márgenes del Guaporé e Iténes, cuando le llegó el encargo de que debían proceder rápidamente, antes de las lluvias (mes de noviembre) a la expulsión de los jesuitas y a la sustitución de éstos por los nuevos curas propuestos por el obispo Francisco Herboso. Se había de vigilar y controlar a los jesuitas a fin de evitar cualquier acto de insubordinación, ocultación o tergiversación de bienes. Por consiguiente Aymerich tenía que atender a varias cuestiones importantes, por un lado al peligro portugués, por otro a la expulsión de los misioneros jesuitas y a la nueva reorganización del pueblo moxo; pero ni militar, ni económicamente estaba preparado. Se estableció en el pueblo de Loreto, el más antiguo y meridional, vecino de Santa Cruz y paso obligado al interior de la zona moxa para enviar desde allí comisarios y piquetes encargados de ejecutar el real decreto (21).

Mientras tanto el superior jesuita, padre Juan de Beingolea, envió a Aymerich una declaración asegurándole la obediencia de los religiosos y su acatamiento a la voluntad real. Con esta declaración Aymerich, el 4 de octubre de 1767 ya posesionado del pueblo de Loreto, previa consigna de todos los bienes por parte de los jesuitas a él y al cura enviado por el obispo Herboso, se dirigía al dicho superior jesuita, residente en San Pedro, para que cursara la correspondiente orden a todas las misiones.

Durante los siguientes meses hasta abril de 1768, los 26 religiosos que había en tierras de moxos fueron llegando a Santa Cruz; José Reyter, cura de Magdalena; Nicolás Sisich y el húngaro Eder, entre otros. En Santa Cruz se unieron a los misioneros de Chiquitos y por Cochabamba llegaron a Tacna y al puerto de Arica.

(21) A. Astrain: Historia de la Compañia de Jesús en la Asistencia de España. Madrid, 1913-25. 
Las misiones después de la Orden de expulsión de 1767 quedaron a cargo directo de la Audiencia de Charcas.

El gobernador don Lázaro de Rivera, sucesor de don Antonio Aymerich y Villajuana, en su informe sobre el Plan o Sistema para el nuevo gobierno de Moxos (1788)(22), nos dice que los jesuitas dejaron quince pueblos «llenos de felicidad y opulencia», habitados por 30.000 almas. En el año de redacción del Informe sólo subsistían once con unos 20.000 habitantes, ya que los indios habían huido en gran número a las fortificaciones brasileñas. Los curas del clero diocesano que se encargaban de las misiones después de 1767, habían masacrado el ganado para lucrarse con el sebo, y aliados de los portugueses les permitieron la entrada para ejercer el contrabando y el robo a cambio de algunas mercancías. Además continúa, «No se hallará en toda la Provincia un cura que sepa de una de estas lenguas, para explicarse en el púlpito, por lo que jamás oyen estos naturales una plática y cuando tienen que auxiliar a alguno van a tartamudear con un cuaderno de oraciones que dejaron los jesuitas".

Por todo ello, Rivera propone que sean reemplazados los curas seglares por subdelegados en el poder espiritual y en el temporal por un administrador secular.

La entrega de los pueblos, en 1767, a la iglesia seglar va a suponer por un lado, que la economía regional pierda su autosuficiencia ante la doble necesidad de importar bienes para contrabandear al Brasil y la utilización como salarios de los productos comunitarios de las misiones. Es decir, se subvertirá el carácter autoabastecedor que los jesuitas tenían implantado en las misiones, convirtiéndolas en fuente de producción para el tráfico intercolonial; hasta entonces la dependencia exterior se había manifestado sólo en la importación de ciertos bienes manufacturados que la Compañía de Jesús conseguía de forma directa en sus propias sedes del mundo colonial, en Madrid o Roma.

Los nuevos usos de la importación hicieron que se diversificara la producción, se depredaran recursos naturales básicos y se agudizara la explotación de la mano de obra indígena, al reducirse el efecto distributivo implantado y base de la economía jesuítica. Este cúmulo de modificaciones fue en detrimento de la población indígena, antes sus productos tropicales y los tejidos de algodón los consignaba a los jesuitas y rendían un beneficio neto al patrimonio de la misión en tanto no eran mal vendidos como lo empezaron a ser por parte de los curas seglares para aumentar

(22) RAH. Colección Mata Linares, IX; fols. 542-563. 
sus arcas personales. Como ya hemos apuntado hasta 1767, las manufacturas eran traídas de Europa directamente por los misioneros y no atravesan la red mercantil que ahora deberían cruzar en su tráfico a Lima.

La vinculación política administrativa con el virreinato del Perú había sido mantenida por los jesuitas en los límites mínimos establecidos por la ley, y desde este punto de vista es lógico pensar que la expulsión de la Compañía y la confiscación de sus tierras significó en la práctica la recuperación por la Corona de una caudalosa fuente de recursos.

Con la confiscación de estas tierras no se formó una élite propitaria, ya que administradas por el clero seglar, no fueron sujetas a apropiación civil ni religiosa. Destruida la red jesuita de reducciones, cada una quedó al arbitrio de curas que en forma individual, abrieron la zona al contrabando y a la penetración económica portuguesa.

\section{LAS BiBliotecas DE LAS MiSIONES DE BuENAVISTA, PAILA Y SANTA ROSA}

\subsection{Misión de Buenavista o Desposorios}

Buenavista fundada en 1723, estaba computada dentro de las misiones de Moxos aunque se encontraba en el cercado de Santa Cruz de la Sierra, a unas catorce o dieciséis leguas de ella. Reducción de indios Yucarés y Churupas - tribu chiquitana que había abandonado siglos atrás su territorio, cerca de Río Grande y se había radicado al oeste de Santa Cruz de la Sierra- al ser éstos los más numerosos el habla de la misión eran la chiquitana; en su momento más álgido se supone que contaba con 2.000 almas, estaba administrada por la Compañía de Lima.

En el momento de la expulsión se encontraban en ella el padre Miguel Rodríguez y el padre Bernabé Seco. En el inventario de la misión realizado en septiembre de 1767, nos describe el escribano el aposento del padre Miguel Rodríguez, cura de ésta: «éste se compone de un estante mediano de libros, dos escritorios, unas petaquillas labradas de suela... Los libros del estante son 
236 (23); además de 19 libros manuscritos de la lengua de las misiones, incluidas el arte de ellas", y en el aposento del padre Bernabé Seco, se encontró un estante con 109 libros, entre grandes y pequeños.

Además la misión contaba con una oficina destinada a carpintería y herrería, con bastantes herramientas; así como almacenes. Casa trapiche, con un gran patio colegio, cocinas, sacristía e iglesia.

En las estancias de la misión: San Joaquín, San Miguel, San Javier, Santa Ana, San Borja, San Ignacio y Dolores se contabilizaron 11.552 cabezas de ganado vacuno 3.790 de ganado caballar, 934 caballos mansos, 37 mulas mansas y 8 chucaras.

La descripción pormenorizada de la librería del aposento del cura Miguel Rodríguez (24) se incluye en Apéndice.

\subsection{Procuración del Puerto de Paila}

La Procuración del puerto de Paila (25), en el camino de Buenavista o Desposorios y Santa Rosa de Sara, estaba computada dentro de las misiones de Moxos, distante unas diez leguas al oriente de Santa Cruz de la Sierra y dieciséis de San Lorenzo de la Barranca (26); fue el único puerto comercial en el río Guaypay, de uso exclusivo de las misiones de moxos y chiquitos, ya que era la única entrada a éstas (en tiempo de lluvias intransitable). Por lo tanto tenía almacenes, estancia para el abasto de las canoas conductoras, dotaciones de acémilas (27) y carretas para el transporte terrestre de manufacturas entre el río y la ciudad. Aquí tenían los jesuitas un colegio, que era hospicio de la Compañía y emporio de efectos de expendio o en tránsito para el Alto Perú. Los portugueses de Mato Grosso traficaban desde los primeros tiempos con la provincia de Santa Cruz de la Sierra por los ríos Iténes, Mamoré y Guaypay o Río Grande hasta el puerto de Paila, considerado como el puerto de Santa Cruz. Era además

(23) AHN. Sección Jesuitas. Leg. 126, n² 22.

(24) Leandro Tormo: "Una biblioteca perdida entre los llanos del Mamoré". En: Missionalia Hispánica (Madrid, 1976), pags. 366-371.

(25) Inventario de la Procuración de Paila (15 de septiembre de 1767), AHN, Sección Jesuitas leg. 126, no 22.

(26) San Lorenzo de la Barranca fue fundada en 1561, pero tuvo que ser trasladada al río Guaypay en 1603-1622, llamándose Santa Cruz de la Sierra.

(27) Mulas/os de carga. 
este colegio un observatorio de los pasos del obispo y del gobierno.

Dicha Procuración, según el inventario hecho en 1767, contaba con: una vivienda, para el padre procurador - Juan Rodríguez (28) - en ella además de tres catres forrados de cuero, ocho sillas, dos mesas con sus cajones vacíos, se encontraba un estante con 91 volúmenes, de ellos 45 libros pequeños de varios títulos, imposible de localizar ya que el autor del inventario no consignó nada más que una leve reseña; que se ofrece íntegra en el Apéndice. Así como toda una serie de materiales: doce cuñas, cuatro machetes, tres azuelas, tres formones, dos escolas, un martillo...

En la vivienda para el mayordomo - Juan Saucedo- donde se hallaron 76 tercios de sal, 10 tercios de hierro con peso de 50 arrobas, con un total de 22 libras, neto; $2 / 3$ de plomo y una sierra grande. Poseían también dos almacenes. En el primer almacén "se desclavó un cajón con el rótulo: $A$ las misiones de Moxos, y en él se halló, 10 tomos de folio del Padre Silveira, 5 tomos medianos de varios títulos, algunos mantos de lana de capullo y dentro de ellos tres potencias de plata para la cruz». Ademas 7 tercios de hierro, platillón; 29 tercios de costales con sus cubiertas, que contiene cada uno 94 costales; 11 de 60 costales y en el centro de cada uno, un costal de lana de capullo de varios colores; 2 costales más de dicha lana de capullo; 7 fanegas de harina de Castilla en 7 costales; 7 petacas de bizcocho de Cochabamba; 1 petaca de suela y en ella muchos talegitos de medallas, botones de senilor y de piedras, gargantillas de piedras de bohemia, rosarios de avalorios engarzados; 1 petaca de suela con 9 piezas de granilla, dos rollitos, atados de cintas batidas y una resma de papel; 1 cajón con dos atados de cuchillos de mesa, uno de tijeras, tres docenas de cuchillos, cuatro de medias de Inglaterra de tercera, coloradas; un cajoncito de granatillos colorados y otras chaquiras de varios colores y una pieza de nácar; 4 petacas de cuchillos de cavo de cuero pintado; $2 / 3$ de plomo; 3 petacas de arroz sin pilas y cuatro cueros de dicho arroz.

En el segundo almacén 55 botijas de vino; 64 tercias de sal, con 8 panes de sal cada tercio.

Por último una casa del administrador interino —José Suá-

(28) El padre Juan Rodríguez llegó a estas misiones relacionado con el Tratado de Límites. La primera reducción que misionó fue en San Miguel (1757), allí fue hecho prisionero por los portugueses junto con el padre Francisco Espi. 
rez-, con dos fondos de cobre, un trapiche, 107 hormas de azúcar que están beneficiando actualmente y los siguientes libros, que se apuntan en Apéndice.

En la capilla una imagen de Nuestra Señora de los Dolores; dos efigies de San Ignacio y San Francisco Javier, una alacena y en ella dos cálices, un platillo y unas vinagreras de plata. Cinco casullas, un alba, tres misales usados, cuatro manteles viejos de altar, dos frontales y una campanita para llamar a la misa a los criados.

La Procuraduría en fin, poseía además 612 cabezas de vacuno, 58 caballos y 46 mulas trajineras y se completaba con la propiedad de doce negros y 20 indios/as de servicio.

\subsection{Misión de Santa Rosa}

La misión de Santa Rosa de Sara, se encontraba en el cercado de Santa Cruz de la Sierra "saliendo del ciudad de Santa Cruz, se caminan doce leguas hasta llegar a un río que llaman Río Grande, en donde se embarca y se sigue su corriente en canoas hasta llegar en cosa de 10 días a otro río más caudaloso que llaman Mamoré cuya corriente contraria se coge y en un día se llega al primer pueblo de Moxos Santa Rosar (29) y a unas doce leguas de Buenavista.

En 1749, el padre Atanasio Teodori, nos dice que contaba con 388 indios, ya que era una fundación reciente.

Poseían una vivienda del cura padre Gabriel Díaz, religioso jesuita y su ayudante padre Juan Estanislao Royo (30). En la vivienda había un estante con 75 libros.

Como la misión estaba recién establecida no se encontraron más papeles ni libros.

Tenía 800 cabezas de ganado vacuno y 100 caballos.

\section{LAS BIBLIOTECAS DE LAS MISIONES}

Los jesuitas por ser la congregación más moderna de implantación en América tuvo una actuación diferente al resto de las

(29) Breve noticia de las Misiones de Mojos en el Obispado de Santa Cruz. de la Sierra en el Reino del Perú (1-5-1776). PASTELl. [2] (1912-49).

(30) Llegó a esta misión en 1763, de 32 años procedente de Casasimarro en el Obispado de Cuenca. 
demás Ordenes, llegando a los dos extremos de la sociedad americana colonial: las élites criollas, y a las masas indígenas. La actuación de la Compañía de Jesús entre los indios fue determinante, a la vez que desarrollaron una actividad económica de expansión en estos territorios, cumplieron una importante función de carácter religioso, educativo y cultural.

Para la labor religiosa, educativa y cultural entre los moxos, los jesuitas mandaron generalmente a éstas misiones a los más preparados para este fin. No todos los que iban a misiones iban a predicar. Los misioneros se distinguieron en la educación de los indios, sacados del bosque y llevados reducciones éstos llegaron a aprender letras, artes manuales, sistemas de construcción, vestidos... hasta la fabricación de bellos instrumentos musicales. En este sistema misional se unían la religión y el estado público, la obediencia y la libertad, el respeto y el amor. Esto se pone de relieve en las pequeñas bibliotecas de estas misiones, y nos ponen al descubierto una faceta claramente valorativa de la actividad en ellas como sería en qué libros, en qué sermones, en qué autores se basan para llevar a cabo esta labor.

Las bibliotecas de las misiones suponen una importante colección de libros, si se tiene en cuenta el lugar y las condiciones ambientales, en ella figuran biblias (B. 5) (31), misales, breviarios (B. 7), sermones, instrucciones de religiosos, letanías, devocionarios, ejercicios espirituales, oraciones, catecismos, obras de teología y moral, hagiografías, filosofía, matemáticas, geografía, agricultura, diccionarios, gramáticas...

Estos libros les servían para ayudarse en sus tareas cotidianas -sermones, libros de oración- o por qué no para pasar e! tiempo leyendo las vidas de santos y misioneros ilustres como las de Jacinto de la Parra (B. 146), Vida de Job (B. 147), Vida del padre Francisco del Castillo (B. 153), Vida del padre Cipriano Barase (B. 154), Vida del padre Alonso Rodríguez (B. 156); o Manuel Bernardes (1644-1710): (P. 2) (32), Meditaciones e historias edificantes (P. 15), La vida de San Francisco de Borja (R. 8) (33).

La relacion del misionero con su medio social es un claro exponente de la cantidad de sermones que poseen en sus bibliotecas, ya que éstos eran el único vehículo eficaz para comunicarse y difundir las ideas y doctrina cristiana, antes de que los indios aprendieran a leer; el púlpito es a la vez lugar de catequesis y

(31) B. Remite a la biblioteca de Buenavista, y el número al de la ficha.

(32) P. Paila.

(33) R. Rosa. 
lugar de lucha ideológica. El sermón en los primeros años fue la ceremonia social de mayor relieve.

Entre los sermones podemos destacar:

En Buenavista: 21 tomos del padre Vieira; varios tomos de Juan de San Gabriel; sermones de Jacinto de Barrasa.

En Santa Rosa: varios tomos del padre Vieira.

El procurador de Paila: 16 tomos en folio del padre Vieira; 3 tomos en 4. ${ }^{\circ}$ del padre Antonio Morín.

El administrador de Paila: 16 tomos en folio del padre Vieira; 9 tomos del padre Antonio Morín; 3 tomos de padre Juan Bautista Masillón; 1 tomo del padre José de Aguilar; 1 tomo del padre Jerónimo de Elso; 2 tomos del padre Salduendo; 1 tomo del Padre San Andrés; 1 tomo del padre Barrasa y un tomo sin autor titulado "Sermones ad populum».

Como se ve los sermones que más abundan son los del padre Vieira jesuita portugués del siglo XVII, célebre predicador se destacó por su denuncia concreta de la situación del indio y del negro. Uno de los postulados esenciales fue que no se permitiera la entrada a los comerciantes a las misiones para evitar el contrabando; estos postulados están íntimamente ligados con la acción de los jesuitas en moxos ya que no permitieron la entrada a comerciantes encomenderos... y fueron guardadas con mucho celo. No es de extrañar que fuera su libro de cabecera.

Los sermones de Vieira están cerca de los del padre Pablo Segneri, encaminados a combatir el vicio y extirpar el pecado entre los indios, pero en el fondo sólo eran lecciones de civismo.

Los padres Elso, Salduendo y Barrasa (criollos peruanos), eran muy proclives a desplegar en sus sermones florituras de retórica muy de la época, ajustándose a la manera de Fray Luis de Granadas, Quevedo o Cicerón, sermones resentidos de culteranismo.

En las trece misiones de Moxos se hablaban siete lenguas distintas, ya que había etnias Moxo, Mobina, Itonama, Guarayo, Caniciana, Cayubaba y otras de clasificación dudosa. Por ello en las bibliotecas las lenguas, es otro de los títulos que más abundan. Junto a los Calepinos como el de Salas (B. 8) aparecen los libros en lengua moxa del padre Pedro Marbán: Arte de la lengua Moxa, con su vocabulario y cathecismo (B. 4), (P. 1) y los vocabularios de lengua chiquita (B. 158), ésta fue estudiada en el siglo XVIII por algunos misioneros jesuitas uno de los más importantes fue el padre Ignacio Chomé (1696-1768), nacido en Dovai. Rehizo y redujo a mejor método lo que habían hecho varios misioneros 
y publicó Vocabulario, tesoro y Arte de la Lengua chiquita, Vocabulario de la lengua zumaca, además tradujo al chiquito varios escritos ascéticos de Nieremberg, redactó un gran diccionario y una historia de la tribu; o los vocabularios en lengua chiriguana (R. 26).

Aparte de los libros en lenguas de los aborígenes, también se encuentran libros en francés como (R. 27): portugés como los del padre Vieira o los del padre Joao da Silveira. La presencia de libros en portugués nos pone de manifiesto la cercanía entre ambos mundos.

Entre los libros litúrgicos destacan: los Breviarios (B. 7), (P. 3), son uno de los libros que no faltan, contienen el rezo eclesiástico de todo el año, excepto de la misa. Antes del siglo XI, los textos usados en el oficio divino estaban contenidos en diversos libros como la Biblia, salterio, antifonario, himnario, sermonario, homiliario. En este siglo apareció por primera vez un volumen único en el que de forma abreviada (de ahí su nombre) estaba reunido el material de los demás. Los primeros breviarios fueron hechos, al parecer para clérigos de la corte pontificia en tiempos de Inocencio III (1160-1216). Los franciscanos divulgaron su uso, y la recitación privada del oficio contribuyó a su difusión. Aunque al principio cada iglesia $u$ orden religiosa confeccionaba su propio breviario, bien que con esquema y fondo comunes, a partir de Pío V (1568) se impuso el Breviario Romano, que sigue en uso aunque ha experimentado varias revisiones.

Los diurnos (B. 30) contienen el rezo eclesiástico de las horas menores, desde laudes hasta completas; los misales, contienen el texto de la misa para todos los días del año y son los que el sacerdote usa en el altar y oficios de misa; los martirologios, libros que contienen la lista oficial y el panegírico de los santos cuya memoria se celebra cada día en la Iglesia Católica.

Los libros blancos (B. 67, 68), llamado así por estar encuadernados con papel blanco, sin imprimir, que sirve para tomar apuntes o notas o para hacer asientos; los libros de bautismo (B. 69), donde se registran los bautismos de la misión; los libros de caja (B. 70) o de registro de la contabilidad donde se inscribe en orden cronológico todas las entradas y salidas de dinero de la Compañía.

Por último haré una mención especial a los libros de medicina como los libros de anatomía (B. 3), cirugía (B. 13), consultas médicas (B. 20), florilegios medicionales, tratados médico-prácticos o la obra de Madame Fouquet (B. 92). 
Los 225 títulos de las bibliotecas que poseían tres pequeñas aldeas jesuíticas de sus misiones de Moxos, en el Alto Perú y en plena frontera con el mundo portugués, representan un altísimo y meritísimo esfuerzo cultural, tanto más elocuente cuanto supone la lejanía y la dificultad de allegarse a aquellas zonas tan marginales.

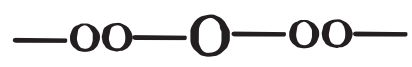




\section{APENDICE}

1. Misión DE BuEnAVISTA. Librería del cura Manuel Rodríguez

1. Herrera, Gabriel Alonso de. Agricultura general / Gabriel Alonso de Herrera. Madrid, 1654 It fol Agricultura general.

2. AMORIS divini 1 t Amoris divinis

3. ANATOMIA 1t Libro de Anatomia.

4. MARBAN, Pedro (S.I.) Arte de la lengua Moxa, con su vocabulario y cathecismo / Pedro Marban. Lima, 1701. 2t de Arte de lengua Moxa.

5. Biblia Sacra. Antuerpia, 1569. It fol Biblia Sacra.

6. Bibloteca Virginalis mariae. Matriti, 1648. 3 vol. fol. $2 t$ fol. Biblioteca virginalis.

7. BREVIARIO. 1t Breviario, viejo. Un Breviario viejo.

8. Salas, Pedro de (S. I.).

Calepino / Pedro de Salas. Madrid, 1762. 1t fol de Calepino de Salas.

9. MESA, Pedro de.

Carta familiar / Pedro de Mesa 1t Carta familiar de don Pedro de Mesa.

10. Francisco de Sales, San.

Cartas Espirituales / San Francisco de Sales. Madrid, 1686. It fol Cartas de San Francisco de Sales. 1 t Cartas espirituales de San Francisco de Sales. 1t Carta de San Francisco de Sales. 6t Vida y cartas espirituales de San Francisco de Sales.

12. Olalla y ARAGON, Frutos Bartolomé.

Ceremonial romano de la misa rezada conforme el misal más moderno, con las advertencias de todo lo que se opone a las Rúbricas para que con toda perfección se ofrezca el Santísimo Sacrificio de la Misa. Frutos Bartolomé Olalla y Aragón. Madrid, 1690. 1 Ceremonial romano.

13. Miro, Magdalena de.

Cirugía / Magdalena Miró. 2t de Cirugía de Magdalena de Miró.

14. Virgilio Maron, Publio.

Comentarios / Publio Virgilio Maron. 1t Comentarios de Virgilio.

15. Comentarios de las epístolas de San Pablo. 1 t fol comentarios de las Epístolas de San Pablo.

16. Concilio o DeCRetos de Trento. 1t Concilio o Decretos de Trento.

17. Concordancias de la Biblia $1 t$ fol. Concordancias de la Biblia.

18. ConCORDIA entre la quietud y la fatiga. It Concordia entre la quietud y la fatiga.

19. CORTES OSSORIO, Juan.

Constancia de la fe y aliento de la nobleza española que escribe y dedica a los gloriosos reinos de Castilla y León / Juan Cortés 
Ossorio. Madrid, 1684. 1t fol Constancia de la fe por el padre Juan Cortes Osorio.

20. Consulta de medicina. 1t Consulta de medicina.

21. Da Religiosa en Soledade. 1t Da religiosa en soledade.

22. Alvarez de PaZ, Diego (S.I.).

De vita espirituali eiusque perfeccione / Diego Jacobo Alvarez de Paz. Maguntiae, 1614. 2v. fol. 1 t fol de Vida Espiritual por el padre Jacobo Alvarez

23. Vega, Pedro de la

Declaración de los siete salmos sentenciales / Padre Pedro de la Vega. Salamanca, 1670. Fol. It fol del Padre Pedro de Vega sobre los Salmos.

24. Declaraciones o decisión novísima del Concilio de Trento. 1t Declaraciones o decisión novísma del Concilio de Trento.

25. DefENSA Canónica. 1t Defensa canonica.

26. Devocio estatu religiosi. 1t Devocio estatu religiosi.

27. Diccionario Pascracio. $2 t$ fol Diccionario Pascracio.

28. DisCreción de las Exequias de la Señora doña M. ${ }^{a}$ Luisa. $1 t$ Discreciòn de las Exequias de la Señora doña M. ${ }^{a}$ Luisa.

29. Solorzano Pereira, Juan de.

Disputatio de Indiarum Jure, sive de justa Indiarum occidentalium Inquisitione, acquisitione et retentiones. Juan de Solórzano Pereira. Matriti, 1653. $2 t$ fol del señor Solórzano de Jure Indiano.

30. DiURnO. 1 Diurno grande.

31. Belarmino, Roberto.

Doctrina Cristiana / Roberto Belarmino. Romae, 1613. Doctrina cristiana por el padre Roberto, $2 t$.

32. IGNACIO DE LOYOLA, San (S.I.)

Ejercicios espirituales / San Ignacio de Loyola. Roma, 1615. It Ejercicios de San Ignacio.

33. Alciato, Andreas.

Emblemata, cum explicationes qua obscura illustrantur, dubiaque omnia soluntur per claudium Minoem / Andreas Alciato. Paris, 1583. It Emblemata Alciata.

34. EMPEÑos del poder y amor de Dios. $1 \mathrm{t}$ fol Empeños del poder y amor de Dios.

35. EPISTOLA gratificatoria. 1t Epistola gratificatoria.

36. Guevara, Antonio.

Epistolas familiares / D. Antonio de Guevara, Obispo de Mondoñedo. Madrid, 1595. 1t Epistolas de don Antonio de Guevara.

37. EsCala Parnasi. 1t Escala parnasi.

38. ESPEJO de verdadera y falsa confesión. 1t Espejo de verdadera y falsa confesión.

39. FIESTAS de la Iglesia Metropolitana de Sevilla. It fiestas de la Iglesia Metropolitana de Sevilla.

40. FLORILEGIO. 1 Florilegio.

41. Flos Sanctoum. 1t fol Flos Sanctorum. 
42. Rivadeneyra, Pedro de (S.I.)

Flos Sactorum o libro de la vidas de los santos. Dirigido a la reina de España doña Margarita de Austria / Pedro de Rivadeneyra. Madrid, 1604.

6t Flos Sactorum por el padre Pedro de Rivadeneyra. En 1761 se editaron 3 vols. aumentados por Juan Eusebio Nieremberg y Francisco García.

43. VILLEGAS, Alonso de.

Flos Sactorum y Historia General de la vida y hechos de Jesu Christo... y de todos los Sanctos de que reza y hace fiesta la Iglesia Católica conforme a Breviario Romano / Alonso de Villegas. Toledo, 1578. It Flox Sactorum por el Maestro Alonso de Villegas.

44. Fuero de la conciencia. 1t Fuero de la conciencia. It Fuero de la conciencia.

45. Fundáción de las Hermanas Descalzas. 1t Fundación de las Hermanas Descalzas.

46. GEOGRAFIA plenior. $2 t$ fol Geografía plenior.

47. GlÓRIAS del segundo siglo. 2 fol Glorias del Segundo Siglo.

48. GriTos del Infierno. 1 t Gritos del Infierno.

49. Guia espiritual. 1t Guía espirutual.

50. Puente, Luis de la (S.I.).

Guía espiritual en que se trata de la oración, imitación y contemplación: de las divinas visitas y gracias extraordinarias: de la mortificación y obras heroicas que la acompañan / Luis de la Puente. Valladolid, 1609. 49. 1t Guia Espiritual por el padre Puente.

51. LOS HECHOS de Alejandro Farnesio. 1t Los Hechos de Alejandro Farnesio.

52. COMPANIÁ DE JESUS.

Historia de la Compañía de Jesús / Compañía de Jesús. It fol Historia de la Compañía de Jesús.

53. Historia de la Florida. $4 t$ fol de la Historia de la Florida.

54. HistORIA de las Guerras Civiles de Francia. 1t Historia de las guerras civiles de Francia.

55. AviLA, Juan de (S.I.)

Historia de la pasión del Hombre-Dios referida y ponderada en décimas españolas / Juan de Avila. León de Francia, 1661. 1t Pasión del hombre Dios por el padre Juan Dávila.

56. CASSANI, José (S.I.)

Historia de la provincia de la Compañía de Jesús del Nuevo Reino de Granada en la América, descripción y relación exacta de sus misiones en el Reino, llanos, mesa y río Orinoco / José Cassani. Madrid, 1741. It fol Historia del Nuevo Reino de Granada.

57. SaAVEDRA FAJARDO, Diego de.

Idea de un príncipe político cristiano, representada en cien mil empreas dedicables al Príncipe de las Españas / Diego de Saavedra Fajardo. Valencia, 1675. 1t Idea de un principe político.

58. FABIO. 
Incarnatu / Fabio. 1t por el padre Fabio incarnatu.

59. INSTITUCIONI Mariani. 1t Institucioni Mariani.

60. CompaÑIA DE JESUS.

Institutum Societatis Jesu / Compañía de Jesús. Pragae: Typis Universitatis Carlo Ferdinandae in colegio Societatis Jesu, 1747. $1 t$ Instituto de la Compañia de Jesús.

61. INSTRUCCION de sacerdotes. 1t Instrucción de sacerdotes.

62. INSTRUCCION sacerdotal. 1t Instrucción sacerdotal.

63. INSTRUCCIONES de religiosos. 1 t instrucciones de religiosos.

64. Granada, Luis de. Introductio del Symbolo de la fe / Fray Luis de Granada. Salamanca, 1585-86. fol. It fol Instrucción de la fe por el padre Fray Luis de Granada.

65. Peña y Montenegro, Alonso de la.

Itinerario para párrocos de Indios / Alonso de la Peña y Montenegro. Madrid, 1668. 1t Itinerario para párrocos de indios.

66. ESCOBAR Y MENDOZA, Antonio (S.I.)

Liber Theologiae moralis viginti quatuor Societatis Jesu. Doctoribus referantum, quem R. P. Antonius de Escobar in examen confessarium digessit add vit illustravit / Antonio Escobar y Mendoza. Lugduni, 1644. 8. 1 t Teologia moral por el padre Antonio Escobar.

67. LIBRo blanco. Un libro blanco con pocas hojas escritas.

68. LIBRo blanco con abecedario. Un libro blanco con abecedario.

69. LIBRo de Bautismo. Un libro de Bautismo antiguo.

70. LIBRO de caja. Un libro de caja.

71. MARTínez DE La PARRA, Juan.

Luz de las verdades católicas y explicación de la doctrina cristiana que siguiendo la costumbre de la casa profesa de la Compañía de Jesús en México, todos los jueves del año ha explicado en su Iglesia / Juan Martínez de la Parra. México, 1691. 1t luz de verdades católicas por el padre Parra.

72. RAMOS, Alonso.

Luz de verdades católicas / Alonso Ramos. It Luz de verdades católicas por el padre Alonso Ramos.

73. MARTIROLOGIO. Un martirologio.

74. MARTIROLOGIO romano. Un Martirologio romano.

75. Misal. Un misal viejo antiguo. Un misal pequeño de a cuartilla, viejo. Un misal de menos de cuarto, viejo. Dos misales de 4o, manuscritos.

76. Agreda, Sor María de Jesús de

Mística ciudad de Dios, milagro de su onmipotencia y abismo de la gracia / Sor María de Jesús de Agreda. Madrid, 1670. 3t fol. Mística ciudad de Dios por la madre Sor María de Jesús. $2 t$ Obras de la madre Agreda.

77. MoRIBUS Subditorum principea. It de Moribus Subditorum principea.

78. NOTACIONES in totas escriturarum sacra. 1t Notaciones in totas escriturarum sacra.

79. Lugo, Juan, Cardenal (S.I.). 
Notas del... cardenal de Lugo sobre los privilegios "Vivae vocis». Oratio del compendio común / Cardenal Juan Lugo. 1t compendio o Notas de Lugo.

80. TORRECILla, Martín de

Obras / Fr. Martín de Torrecilla. 7t fol Obras de Torresillas.

81. Quintana Dueñas, Antonio (S.I.)

Obras / Antonio Quintana Dueñas. 2t fol. Obras del padre Quintana.

82. SilveIRA, Joao da.

Obras / Joao de Silveira. $2 t$ fol del padre Silveira. $4 t$ fol, Obras del padre Silveira.

83. TAMBURINI, Tomás (S.I.)

Obras / Tomas Tamburini. 5t fol Obras del padre Tomás Tamburini.

84. Moya, Mateo de.

Obras / Mateo de Moya, pseudónimo de Lomarencis Amadeus Guimenius. $2 t$ fol Obras del padre Mateo de Moya.

85. Puente, Luis de la (S.I.)

Obras / Luis de la Puente. $2 t$ fol Obras del padre Puente.

86. Caussino, Nicolás (S.I.)

Obras / Nicolás Caussino. Madrid, 1677. 4t fol Obras del padre Nicolás Causino.

87. RivadenEYRA, Pedro de (S.I.)

Obras / Pedro de Rivadeneyra. It fol Obras del padre Pedro de Rivadeneira. 1t del dicho autor Rivadeneira.

89. TERESA DE JESUS, Santa.

Obras / Santa Teresa de Jesús. Amberes, 1649-1661. 5t Obras de Santa Teresa.

90. Virgilio Marón, Publio.

Obras en castellano / Publio Virgilio Maron 1t Obras de Virgilio Maron en castellano.

91. BILlEN DE BIEDMA, Dr.

Obras líricas / Doctor Billen de Biedma. 1t fol. Obras líricas por el doctor Billen de Biedma.

92. FOUQUET, Madame.

Obras médico-quirurgicas / Madame Fouquet. Valladolid, 1748. It Medicina de Madame Foquet.

93. BAGLIVI.

Opera omnia médico-práctica et anatómica / Baglivi. Lugduni, 1704. 1t Medicina Baglivi.

94. SEGNERI, Paolo (S.I.)

Opere / Paolo Segeneri. Parma, 1701. 16t Obras del padre Segneri.

95. NIEREMBERG, Juan Eusebio (S.I.)

Opere spirituale /Juan Eusebio Nieremberg. Venezia, 1715. $2 t$ fol. Obras espirituales del padre Nieremberg.

96. EL OYENTE remediado. $3 t$ El oyente remediado.

97. GARZONI, Tommaso.

Plaza Universal de todas las ciencias y artes. Traducido al castellano 
por Cristóbal Suárez de Figueroa / Tommaso Garzoni. Madrid, 1615. 1t Plaza universal de las ciencias.

98. LaNGIO, Joseph.

Poliantea Novissime / Joseph Langio. Francofurti, 1607. 1t fol Novisima polianthea.

99. Castillo de Bovadilla, Jerónimo.

Política para corregidores y señores vasallos, en tiempos de paz y de guerra, y para jueces ecclesiásticos y seglares y para regidores y abogados / Jerónimo Castillo de Bovadilla. Madrid, 1597. 2 vols. fol. It fol de la Política de Bobadilla.

100. Práctica y defensa de la inmunidad eclesiástica. It Práctica y defensa de la inmunidad eclesiastica.

101. Recopilación de las leyes de Indias. Madrid, 1640. 4t fol de la Recopilación de Indias.

102. RELACIón de las exequias de la señora doña María Luisa de Borbón. 1t Relación de las exequias de la señora doña María Luisa de Borbón.

103. REPAROS Historiales. 12t Reparos Historiales.

104. RITUAL romano. 1t Ritual Romano.

105. Salmos del Nuevo Testamento. 8 libritos de Salmos del Nuevo Testamento.

106. SeCRETos de agricultura. 1t de Secretos de Agricultura. $2 t$ dichos, sin cubiertas, ni principio ni fin.

107. Selectarum epistolarum. 1t Selectarum epistolarum.

108. VIEIRA, Antonio (S.I.)

Sermones / Antonio Vieira. Madrid, 1711. 21 vols. 21t del padre Vieira Sermones.

109. SAN GABRIEL, Juan de.

Sermones sobre los evangelios de Domingos, Miércoles y Viernes de Quaresma. Fr. Juan de San Gabriel. Sevilla, 1643. 3 vols. 4. It Sermones del padre Fr. Juan de San Gabriel.

110. BARRASA, Jacinto.

Sermones varios predicables... en el reyno del Perú / Jacinto Barrasa. Madrid, 1678. It Sermones del padre Barrasa.

111. LACROIX, Claudio.

Sin título / Claudio Lacroix. $2 t$ fol. del padre Lacroix.

112. RAmirez, André Pinto (S.I.)

Sin título / Andrés Pinto Ramírez. 4t fol. del padre Pinto Ramírez. It fol. del padre Pinto Ramirez.

113. DREXEL, Hieremias (S.I.)

Sin título / Hieremias Drexel. 2t. fol del padre Drexeley.

114. LEÓN MAGNO, San.

Sin título / San León Magno. 1 t fol. de San León Magno.

115. AvilA, Juan de (S.I.).

Sin título / Juan de Avila. It fol. del padre Juan de Avila.

116. Lugo, Juan, Cardenal (S.I.).

Sin título / Cardenal Juan Lugo. It fol. del Cardenal Lugo. 
117. TAMBURINI, Tomás (S.I.)

Sin título / Tomás Tamburini. 1 fol del padre Tomás Tamburino.

118. MACHADO.

Sin título / Machado. 4t fol del padre Machado.

119. Villalobos, Enrique de.

Sin título / Enrique de Villalobos. $2 t$ fol de Villalobos.

120. NATALI, Juan.

Sin título / Juan Natali. It por el padre Juan Natali.

121. BataBI, Dionisio.

Sin título / Dionisio Batabi. 2t Dionisio Batabi.

122. Olalla y Aragón, Frutos Bartolomé.

Sin título / Frutos Bartolomé. Olalla y Aragón. 1 t del padre Olalla.

123. Penequín, Pedro.

Sin título / Padre Pedro Penequín. 1t por el Padre Pedro Penequín.

124. MolinA; Gregorio de.

Sin título / Dr. Gregorio de Molina. 1t del Dr. Gregorio de Molina.

125. HOMERO.

Sin título / Homero. 1 libro de Homero en romance.

126. SALAS, Pedro de (S.I.).

Sin título / Pedro de Salas. 1t por el padre Pedro de Salas.

127. Ovidio Nason, Publio.

Sin título / Publio Ovidio Nason. En latín. 1t Ovidio latino.

128. LESSIUS, Leonard Leys (S.I.)

Sin título / Leonard Leys Lessius.1t de Leonardo Lesso.

129. LARRAGA, Francisco.

Sin título / Francisco Larraga. 1t por el padre Larraga.

130. Rocha, Diego Andrés.

Sin título / Diego Andrés Rocha. 1t por el padre Andrearocha.

131. BaLTASAR, Juan Antonio (S.I.).

Sin título / Juan Antonio Baltasar. 1t por el padre Baltasar.

132. NÁJERA, Manuel.

Sin título / Manuel Nájera. 1t por èl padre Manuel Nájera.

133. BERNARDI, Teofixi.

Sin título / Teofixi Bernardi. 1t Teofoxi Bernardi.

134. Sin título. 2 libros viejos sin pergamino. 1 libro viejo. 3 libros de la lengua manuscritos. 1 libro de cuentas de la misión con otro pequeño de las estancias. 107 libritos de media cuartilla, algunos menos y otros sin pergamino, con la advertencia que todos están maltratados.

135. TORRECILLA, Martín de.

Suma de todas las materias morales / Fr. Martín de Torrecilla. Madrid, 1693. 2 vol. $2 t$ fol Suma del Padre Torrecilla.

136. RICHARDO, Antonio.

Teología / Antonio Richardo. 3t de Teología del padre Richarde.

137. Plasentino, Francisco.

Teología Moral / Plasentino, Francisco. $2 t$ fol. Teología Moral por el padre Francisco Plasentino o Pascutino.

138. TESORO de la Biblia. 1t Tesoro de la Biblia. 
139. Ovidio Nason, Publio.

Transformaciones / Publio Ovidio Nason. 1t Transformaciones de Ovidio.

140. Alvarez de Paz, Diego (S.I.)

Tratado de los mandamientos de la Iglesia / Diego Alvarez de Paz. 1t Tratado de los mandamientos de la Iglesia por el padre Diego Alvarez.

141. Bustamente, Juan de.

Tratado del oficio divino / Padre Juan de Bustamante. Madrid, 1659. 1t 4o Tratado del oficio divino por el padre Juan de Bustamante.

142. TRATADO para saber rezar bien el oficio divino. 1t Tratado para saber rezar bien el oficio divino.

143. SEGNERI, Paolo (S.I.)

Tratado para saber rezar bien el oficio divino / Paolo Segeneri. $1 t$ Tratado para saber rezar bien el oficio divino por el padre Señeri.

144. VERDADEROS entretenimientos. 1t Verdaderos entretenimientos.

145. VIA vite eterni. It via vite eterni.

146. PARRA, Jacinto de la.

Vida admirable y muerte preciosa de la venerable madre sor Rosa de Santa María Peruana / Fr. Jacinto de la Parra. Valencia, 1665. $1 t$ fol. Vida de Santa Rosa.

147. VIDA de Job. It Vida de Job o Sab.

148. ViDA de Juan de Allosa. It Vida de Juan de Allosa.

149. VIDA de la venerable madre sor Juana Francisca. It Vida de la venerable madre sor Juana Francisca.

150. VIDA de San Estanislao. 1t Vida de San Estanislao.

151. TURSELINO, Horacio (S.I.)

Vida del P. Francisco Javier de la Compañía de Jesús, escrita en latín por el padre Horacio Turselino y traducida por el padre Pedro de Guzmán, natural de Avila, religiosos de la misma compañía / Horacio Turselino. Valladolid, 1600. 1t Vida de San Francisco Javier. Vida de San Francisco Javier 1 t.

152. VIDA de San Ignacio. It Vida de San Ignacio. 1t. Vida de San Ignacio.

153. VIDA del padre Francisco del Castillo. 1t Vida del padre Francisco del Castillo.

154. VIDA del reverendo padre y venerable Cipriano Barese. It Vida del reverendo padre y venerable Cipriano Barese.

155. FRANCISCO DE SALES, San.

Vida simbólica / San Francisco de Sales. Madrid, 1688. 6t Vida y Cartas espirituales de San Francisco de Sales.

156. Colin, Francisco.

Vida, hechos y doctrina del venerable hermano Alonso Rodríguez de la Compañía de Jesús / Francisco Colin. Madrid, 1654. 4². It Vida del venerable Alonso Rodriguez.

157. NeBrija, Antonio de.

Vocabulario Antoniano / Antonio de Nebrija. León de Francia, 1655. It Vocabulario antoniano. 
158. Vocabularios de la lengua chiquita. 2 Vocabularios de la lengua chiquita.

\section{Procuración del puerto de Paila}

\subsection{Libros del padre procurador}

1. FERRARIS, Martín.

Biblioteca canónica, jurídica, moralis, theológica / Martín Ferraris. Roma, 1758-62. 5 t. fol Biblioteca Moralis.

2. BERNARDES, Manoel.

Exercicios espirituales e meditaçoes / P. Manoel Bernardes. Lisboa, 1706. 10t. $4^{\circ}$ Meditaciones de Manuel Bernardez.

3. TIRINUS, Jacobi.

In sacram scripturam / Jacobi Tirinus. Lugduni, 1702. 2 vols. fol. $2 t$ fol. P. Thirini Comentarios Sacra escriture.

4. Medalla evangélica. $7 t 4^{\circ}$ Medalla evangélica.

5. MORín, Antonio.

Sermones / Antonio Morín. $3 t 4^{\circ}$ Sermones del Padre Antonio Morín.

6. VIEIRA, Antonio (S.I.)

Sermones varios / Antonio Vieira. Madrid, 1711. 2v. 16 t. fol Sermones del padre Vieira.

7. Sin título. 45 libros pequeños de varios títulos.

8. BELAMino, Roberto.

Universis partis / Roberto Belarmino. $2 t$ fol. Roberti Universis partis.

\subsection{Libros del administrador}

1. Marban, Pedro (S.I.).

Arte de lengua moxa, con su vocabulario y cathecismo / Pedro Marban. Lima, 1701. 1t Arte de la lengua moxa.

2. BiBloteCA Moralis. $5 t$ fol. Biblioteca Moralis.

3. BREVIARIO evangélico. $2 t$ Breviario evangelico.

4. COMENTARIOS in Sacra Theologia. 3t Comentarios in Sacra Theologia.

5. CONTEMPLACIS moralis. 1t Contemplacis Moralis.

6. SEGNERI, Paolo (S.I.).

El cristiano instruido en su ley; discursos morales y doctrinales; traducidos del toscano por Espinola Baeza / Paolo Segneri. Barcelona, 1693. 1t Cristiano instruido.

7. Devoto de María. It Devoto de María.

8. EJERCICIOS espirituales. It Ejercicios espirituales.

9. FRANCISCO JAVIER, San.

Epistolarum libri quattuor / San Francisco Javier. 1t Epistolas de San Javier.

10. TIRINUS, Jacobi. 
In sacram scripturam /Jacobi Tirinus. Lugduni, 1702. 2 vols. fol. $2 t$ Tirini Comentarios sacra escritura.

11. LiBRos de varios títulos. 4t de varios títulos. 4t descuadernados y un manuscrito.

12. Medalla evangélica. Un juego de $7 t$ Medalla evangélica.

13. MERCURIO. 1t Mercurio.

14. ORACIONES. 1t Oraciones, it pequeño.

15. Bernardes, Manoel. Pasto /Manoel Bernardes. 1t Bernardes Pasto.

16. ReloX cristiano. 1t Relox cristiano.

17. MORIN, Antonio.

Sermones / Antonio Morin. 6t Sermones del padre Antonio Morin. 3t Sermones del padre Antonio Morin.

18. SAN ANDRÉS.

Sermones / Padre San Andrés. 1t Sermones del padre San Andrés.

19. SERMONES ad populum. 1t Sermones ad populum.

20. SALDUENDO, Francisco Javier (S.I.).

Sermones morales / Francisco Javier Salduendo. Madrid, 1716. 1t Sermones morales del padre Salduendo.

21. MASILlON, Juan Bautista.

Sermones sobre diferentes evangelios de Quaresma / Juan Bautista Masillon. Madrid, 1730. 3v. 4. 3t Masillon Sermones evangelicos.

22. VIEIRA, Antonio (S.I.)

Sermones varios / Antonio Vieira. Madrid, 1711. 21 vols. $16 \mathrm{t}$ fol. del padre Vieira.

23. SALDUENDO, Francisco Javier (S.I.).

Sermones varios / Francisco Javier de Salduendo. Madrid, 1716. 1t Sermones del padre Salduendo.

24. BARRASA, Jacinto.

Sermones varios predicados en el reino del Perú / Jacinto Barrasa. Madrid, 1678. 1t Sermones del padre Barrasa.

25. Aguilar, José de.

Sermones varios predicados en la ciudad de Lima / José de Aguilar. Bruselas, 1684. 1t Sermones de Aguilar.

26. ElSo, Jerónimo de (S.I.)

Sermones varios, obra phostuma del ... de la $\mathrm{C}$. ${ }^{a}$ de Jesús en la provincia de Lima, que da a luz su fiel amigo don Diego Portolés Meneses / Jerónimo de Elso. Madrid, 1731. It Sermone del padre Elso.

27. Segneri, Paolo (S.I.).

Cristiano instruido en su ley / Paolo Segneri. Madrid, 1694. Un juego de 10t del padre Segneri.

28. MUNIESA.

Sin título / Muniesa. It del padre Muniesa.

29. SILVEIRA.

Sin título / Silveira. 10t del padre Silveira.

30. ILLSUMG, Jacobo.

Teología Moral / Jacobo Illsumg. It Teologia Moral del padre Jacobo Illsumg. 
31. BELARMino, Roberto.

Universis Partis / Roberto Belarmino. 2t Roberti Universis Partis.

32. VERDADES católicas. $2 t$ Verdades católicas.

\section{Misión de SANTA Rosa}

1. MARTínez. Biblia philologica / Martínez. 1t Biblia philologica de Martinez.

2. CARTAS edificantes. $16 t$ Cartas edificantes.

3. TAPIA, Pedro de. Cathenae moralis doctrina / Pedro de Tapia. Sevilla, 1654-57. $3 t$ Cathanea.

4. Murillo Velarde, Pedro.

Cursus Juris Cononii Hispani, et Indice in qua juxta ordinen titulorum Decretalium non solum canonice decisiones offeruntur, sed in super additur, quod in nostro Hispaniae Regno... / Pedro Murillo Velarde. Madrid, 1743. 2 v. fol. $2 t$ Murillo Derecho canonico.

5. LOSSADA, Luis de.

Cursus philosophici, regalis collegi Salmanticae Societatis Jesu in compendium redacti et in tres partes divisi / Luis de Lossada. Salamanca, 1730-35. Losada Curso Filosofico $4 t$.

6. DE VITIS, patrum. 1t De vitis patrum.

7. TURSELINO, Horacio.

Epitome historiaum / Horacio Turselino. 1t Turselino Epitome Historiarum.

8. Cienfuegos, Alvaro.

La heroica vida, virtudes y milagros del grande San Francisco de Borja / Alvaro de Cienfuegos. Madrid, 1702. 1t Cientuegos Vida del padre Borja.

9. BUSSIERES.

Historia de Francia / Bussieres. 4t Busieres Historia de Francia.

10. LIBROS. 22t pequeños.

11. BuSEBAUM, Hermano (S.I.)

Médula de la Theologia moral, que con fácil y claro estilo explica y resuelve sus materias y casos / Hermano Busebaum. Zaragoza, 1664. 1t Medulla de Busembau.

12. RETÓRICA. 2t Retórica.

13. RITUAL mexicano. 1t Ritual mexicano.

14. TARQUINO.

Segundo tomo de oraciones / Tarquino. 1t de Tharquino, el segundo de oraciones.

15. MONTENEGRO.

Sin título / Montenegro. 1t Montenegro.

16. LACROIX, Claudio.

Sin título / Claudio Lacroix. 2t Lacrois.

17. MaChADO.

Sin título / Machado. 2t Machado. 
18. Torrecilla, Martín de.

Sin título / Martín de Torrecilla. $2 t$ Torrecilla.

19. SÁNCHEZ.

Sin título / Sánchez. 5t Sanchez.

20. VIEIRA, Antonio (S.I.).

Sin título / Antonio Vieira. $2 t$ Vieira.

21. TACiTo, Cayo Cornelio.

Sin título / Cayo Cornelio Tacito. 1t Tacito.

22. MARCIAL, Marco Valerio.

Sin título / Marco Valerio Marcial. 1t Marcial.

23. JUVENAL.

Sin título / Juvenal. 1t Juvenal.

24. Curcio Rufo, Quinto.

Sin título / Quinto Curcio Rufo. 1t Quinto Curcio, en francés.

25. VIDA de Santa Teresa. 1t Vida de Santa Teresa.

26. VOCABUlARIO de lengua chiriguana. $4 t$ de lengua chiriguana.

27. VOCABULARIO francés. 1t Vocabulario francés. 